\title{
The Inheritance of Dental Traits in a Chinese Population in the United Kingdom
}

\author{
G. T. R. LEE and D. H. GOOSE \\ School of Dental Surgery, University of Liverpool
}

Dental traits such as those of Carabelli or incisor shovelling have fascinated anthropologists and dentists for many years. This is probably because they are easily observed in both living and skeletal material and they show ethnic differences in frequency which might have a genetic basis.

If, however, these traits are to be of any value their mode of inheritance must be understood and so some preliminary work was done on an existing Liverpool family study described by Bowden and Goose (1969). In this material the behaviour of one trait (Carabelli's) was followed. This trait is situated on the lingual aspect of the mesio-lingual cusp of the maxillary 1st and, to a lesser extent, 2nd and 3rd molars (Fig. 1). It may take the form of a pit, fissure, or cusp, and following Kraus (1951), 4 categories are noted; (a) no evidence of Carabelli's trait-smooth surface with the absence of pits or fissures, (b) pits or fissures, $\left(c_{1}\right)$ cusp without free tip, and $\left(c_{2}\right)$ cusp with free tip.

It was found that the formerly suggested model of 2 allelic autosomal genes without dominance did not seem satisfactory and it was suggested that the inheritance might be multifactorial (Goose and Lee, 1971).

Further dental traits were then examined but owing to the large number of missing or filled teeth in the parents no fully satisfactory data could be obtained. However another population (Chinese) was available in Liverpool which it was felt might not suffer so much from this problem and so it was decided to investigate them as well.

\section{Materials and Methods}

One hundred of these Chinese families living in Liverpool and the surrounding districts who were either known personally or through contacts were visited. The average age of the parents was 38 with a range of between 29 and 55 years whilst the average age of the children was 12 with a range of 7 to 22 years. Nearly all the parents were immigrants, only 5 being born

Received 29 February 1972. locally. On the other hand over $70 \%$ of the children were born in the United Kingdom.

The families were visited at their homes and dental impressions of the upper and lower teeth were taken using an accurate impression material. The impressions were cast as soon as possible using a high grade dental plaster. From the casts produced the following observations were made.

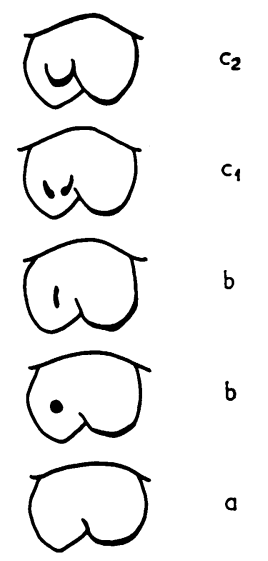

FIg. 1. Carabelli's trait. Cusp with free tip $\left(\mathrm{C}_{2}\right)$, cusp without free tip $\left(C_{1}\right)$, groove and pit $(b)$, and no evidence $(a)$.

Shovelling of Incisors. The term 'shovelshaped' refers to a combination of a concave lingual surface and elevated marginal ridges enclosing a central fossa in incisor teeth (Fig. 2). Hrdlicka (1920) classified maxillary incisors into 4 categories; (a) shovel-enamel rim distinct with the enclosed fossa well developed; (b) semi-shovel-enamel rim distinct but enclosed fossa shallow; (c) trace-shovel-traces of enamel rim which could not be classed as semi-shovel; (d) no shovel-no perceptible trace of rim or fossa.

Maxillary Molar Cusp Number. Generally the maxillary first molar exhibits 4 cusps but the 2 nd and 3rd molars are more variable due to evolutionary reduction leading to the diminution or elimination of the distolingual cusp (Fig. 3). In the present study only the 2nd molar has been studied and 3 categories have been used; 4-cusped teeth (4), distolingual cusp reduced in size 

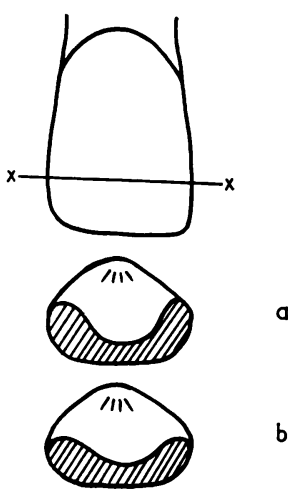

b
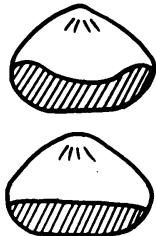

Fig. 2. Shovel-shaped incisors. Section through X-X. Shovel (a), semi-shovel (b), trace-shovel (c), and no shovel (d).

$(3+)$, and absence of the distolingual cusp (3) (Turner, 1969).

Mandibular Cusp Number and Fissure Pattern (Fig. 4). The occlusal form of mandibular molars in man may vary from the primitive 5-cusp 'dryopithecus' pattern bearing the $\mathrm{Y}$ fissure configuration to extreme modification of 4 cusps with an $\mathrm{X}$-shaped configuration. The $\mathrm{Y}$ fissure configuration shows contact of the mesiolingual cusp with the middle buccal cusp. The + pattern shows a cruciform fissure configuration where there is a point contact between the 4 main cusps. In the present study the mandibular 2 nd molar only was classified into 4- or 5-cusped and $\mathrm{Y},+$, or $\mathrm{X}$ fissure configuration since the 1st was often absent or obscured by fillings.
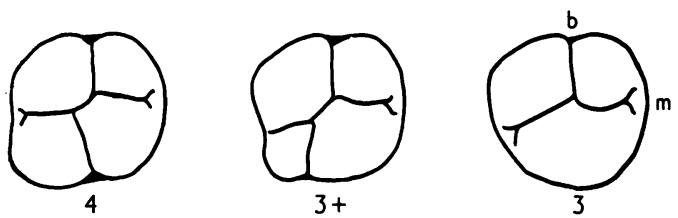

FIG. 3. Maxillary molar cusps. Four cusps (4), reduced distolingual cusp $(3+)$, and 3 cusps ( 3$) ; b=$ buccal, $m=$ mesial.

\section{Results}

Kraus (1951) suggested that for Carabelli's trait one homozygote showed no feature (cc), the heterozygote pits or grooves $(\mathrm{Cc})$, and the other homozygote a cusp (CC). In Fig. 1 these are labelled a, b, and $c_{1}$ and $c_{2}$, respectively. Table I shows the offspring from different mating types.

TABLE I

OFFSPRING WITH CARABELLI'S

TRAIT (FIRST MOLAR) PRODUCED BY DIFFERENT MATINGS

\begin{tabular}{l|r|r|c}
\hline \multirow{3}{*}{ Matings } & \multicolumn{3}{|c}{ Offspring } \\
\cline { 2 - 4 } & $\mathrm{cc}$ & $\mathrm{Cc}$ & $\mathrm{CC}$ \\
\hline $\mathrm{cc} \times \mathrm{cc}$ & 22 & 30 & 0 \\
$\mathrm{cc} \times \mathrm{Cc}$ & 5 & 25 & 2 \\
$\mathrm{cc} \times \mathrm{CC}$ & 3 & 7 & 2 \\
$\mathrm{Cc} \times \mathrm{Cc}$ & 2 & 10 & 0 \\
$\mathrm{Cc} \times \mathrm{CC}$ & 0 & 0 & 0 \\
$\mathrm{CC} \times \mathrm{CC}$ & 0 & 0 & 0 \\
\hline
\end{tabular}

$c c=a, C c=b$, and $C C=c_{1}+c_{2}($ see Fig. 1)

TABLE II

OFFSPRING WITH SHOVEL-SHAPED INCISORS PRODUCED BY DIFFERENT MATINGS

Central Incisor

\begin{tabular}{|c|c|c|c|}
\hline \multirow{2}{*}{ Matings } & \multicolumn{3}{|c|}{ Offspring } \\
\hline & SS & Ss & ss \\
\hline $\begin{array}{l}\mathbf{S S} \times \mathbf{S S} \\
\mathbf{S S} \times \mathbf{S s}\end{array}$ & $\begin{array}{l}38 \\
38\end{array}$ & $\begin{array}{r}4 \\
26\end{array}$ & $\begin{array}{l}0 \\
0\end{array}$ \\
\hline \multicolumn{4}{|c|}{ Lateral Incisor } \\
\hline \multirow{2}{*}{ Matings } & \multicolumn{3}{|c|}{ Offspring } \\
\hline & SS & Ss & ss \\
\hline $\begin{array}{l}\mathbf{S S} \times \mathbf{S S} \\
\mathbf{S S} \times \mathbf{S s} \\
\mathbf{S S} \times \mathbf{S S} \\
\mathbf{S s} \times \mathbf{S s}\end{array}$ & $\begin{array}{r}16 \\
16 \\
0 \\
1\end{array}$ & $\begin{array}{r}4 \\
21 \\
0 \\
4\end{array}$ & $\begin{array}{l}0 \\
2 \\
0 \\
0\end{array}$ \\
\hline
\end{tabular}

SS $=a$, Ss $=b$, and $s s=c+d($ see Fig. 2$)$

Turner (1969) suggested in his Eskimo study that in the case of shovelling one homozygote showed no feature or just a trace (ss), the heterozygote, semishovel (Ss), and the other homozygote full shovel (SS). In Fig. 2 these are respectively $c$ and d, b and a. He also stated that in maxillary molar cusp number one homozygote showed 4 cusps ( $\mathrm{HH})$, the heterozygote a reduced cusp ( $\mathrm{Hh}$ ), and the other homozygote 3 cusps (hh). In Fig. 3 these are labelled $4,3+$, and 3 respectively. The matings
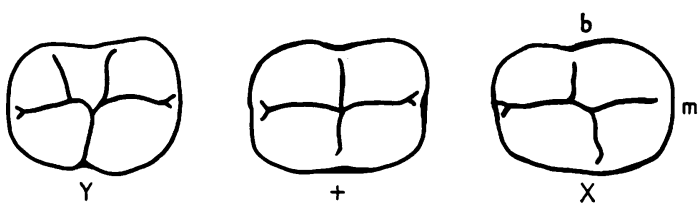

Fig. 4. Mandibular molar fissure configuration. $\mathbf{Y},+$, and $\mathbf{X}$ patterns; $b=$ buccal, $m=$ mesial. 
TABLE III

OFFSPRING WITH DIFFERENT

MAXILLARY MOLAR CUSP

NUMBERS (SECOND MOLAR)

PRODUCED BY DIFFERENT MATINGS

\begin{tabular}{c|c|c|c}
\hline \multirow{2}{*}{ Matings } & \multicolumn{3}{|c}{ Offspring } \\
\cline { 2 - 4 } & hh & Hh & HH \\
\hline hh $\times$ hh & 2 & 1 & 1 \\
hh $\times$ Hh & 0 & 0 & 0 \\
Hh $\times$ Hh & 0 & 0 & 3 \\
hh $\times$ HH & 14 & 1 & 4 \\
Hh $\times$ HH & 0 & 1 & 2 \\
HH $\times$ HH & 1 & 0 & 12 \\
\hline
\end{tabular}

$\mathrm{hh}=3, \mathrm{Hh}=3+$, and $\mathrm{HH}=4$ (see Fig. 3 )

for these two characters are shown in Tables II and III.

By analogy the same type of model is tested here for lower molar fissure pattern, $\mathrm{Y}$ being one homozygote (YY), + being the heterozygote (Yy), and $X$ the other homozygote (yy) (Fig. 4). These matings are shown in Table IV.

TABLE IV

OFFSPRING WITH DIFFERENT

MANDIBULAR MOLAR FISSURE

CONFIGURATION (SECOND MOLAR)

PRODUCED BY DIFFERENT MATINGS

\begin{tabular}{c|c|c|c}
\hline \multirow{2}{*}{ Matings } & \multicolumn{3}{|c}{ Offspring } \\
\cline { 2 - 4 } & YY & Yy & yy \\
\hline YY $\times$ YY & 0 & 0 & 0 \\
YY $\times$ Yy & 0 & 0 & 0 \\
YY $\times$ yy & 0 & 3 & 1 \\
Yy $\times$ Yy & 0 & 1 & 1 \\
Yy $\times$ yy & 0 & 5 & 3 \\
yy $\times$ yy & 3 & 7 & 3 \\
\hline
\end{tabular}

$\mathrm{YY}=\mathrm{Y}, \mathbf{Y} \mathrm{y}=+$, and $\mathrm{y} \mathbf{y}=\mathbf{X}$ (see Fig. 4)

Only 4- or 5-cusped lower 2nd molars were available in the series and thus the previous hypotheses would not be possible in this case. An attempt was made to test a simple dominant recessive model but the result was inconclusive.

\section{Discussion}

In the past it has been hoped that such characters being easily observed might be useful in genetic analysis; it is therefore important to know what hereditary mechanisms are involved. Examination of the Tables reveals many discrepancies from that expected from the proposed model. For example in Table II, 4 heterozygotes are produced from like homozygote matings in the case of the central and lateral incisors and 2 homozygotes (ss) from the mating of the other homozygotes (SS) with heterozygotes. In Table I, 30 heterozygotes are produced from the mating of like homozygotes, 2 homozygotes (CC) are produced from matings of the other homozygotes (cc) and heterozygotes; homozygotes are also produced by matings of the 2 unlike homozygotes.

In Table III mating of like homozygotes (hh) produce a heterozygote and the other homozygote, matings of unlike homozygotes produce 18 homozygotes ( 14 of one and 4 of the other). In Table IV, one homozygote (yy) is produced from matings of unlike homozygotes and 3 homozygotes (YY) and 7 heterozygotes from matings of the other homozygotes (yy).

Although certain upsets in mating patterns might be due to misclassification or illegitimacy the numerous exceptions to the 4 codominant models seem conclusive proof that this is not the correct hypothesis.

In the case of the mandibular molar cusp number there is insufficient evidence to make any comment about the adequacy of the dominant recessive model and it would be interesting to see some more data on this.

\section{TABLE V}

DIFFERENT TYPES OF CARABELLI MANIFESTATIONS PRODUCED BY DIFFERENT MATINGS IN CAUCASAIN FAMILIES (GOOSE AND LEE, 1971)

\begin{tabular}{l|c|c|c}
\hline \multirow{2}{*}{ Matings } & \multicolumn{3}{|c}{ Offspring } \\
\cline { 2 - 4 } & CC & Cc & cc \\
\hline $\mathrm{cc} \times \mathrm{cc}$ & 0 & 2 & 0 \\
$\mathrm{cc} \times \mathrm{Cc}$ & 5 & 6 & 4 \\
$\mathrm{cc} \times \mathrm{CC}$ & 1 & 1 & 2 \\
$\mathrm{Cc} \times \mathrm{Cc}$ & 2 & 7 & 0 \\
$\mathrm{Cc} \times \mathrm{CC}$ & 0 & 2 & 0 \\
$\mathrm{CC} \times \mathrm{CC}$ & 0 & 0 & 0 \\
\hline
\end{tabular}

Taken as a whole, therefore, simple Mendelian models seem unacceptable for these common dental traits and it appears necessary to look again carefully at them. All have been classified into groups but not always in the same way. For example Dahlberg (1963) described 7 manifestations of the Carabelli trait, and even then intermediate forms were present. Incisor shovelling has even been measured metrically, for example the depth of the lingual fossa in mm (Carbonell, 1963) and thus the traits are better regarded as variates taking any value between limits rather than attributes in a statistical sense. (They have merely been called attributes for convenience of classification.)

It appears therefore that it would make better sense to assume these traits are really continuous and not discrete and therefore are likely to be inherited in a multifactorial way. This confirms the findings of Goose and Lee (1971) in connection with 
Carabelli's trait in a Caucasoid population and their results are given in Table $\mathrm{V}$.

\section{Summary}

Dental impressions were taken from 100 Chinese families in Liverpool and the resulting models examined for 5 common dental traits: shovelling of incisors, Carabelli's trait, maxillary molar cusp number, mandibular molar cusp number, and fissure patterns.

The offspring of the various mating types for each trait were tabulated and tested on the assumption of a codominant model in the first 4 and a dominantrecessive one for the fifth.

There were wide discrepancies shown and it was concluded that simple Mendelian inheritance is unlikely in these traits. More probably a multifactorial system is applicable.
We would like to thank the Medical Research Council for the grant by which this work was undertaken.

\section{REFERENCES}

Bowden, D. E. J. and Goose, D. H. (1969). Inheritance of tooth size in Liverpool families. Fournal of Medical Genetics, 6, 55-58.

Carbonell, V. M. (1963). Variation in the frequency of shovelshaped incisors in different populations. In Dental Anthropology, ed. by D. R. Brothwell. 5th edition. Pergamon Press, Oxford.

Dahlberg, A. A. (1963). Analysis of the American Indian dentition. In Dental Anthropology, ed. by D. R. Brothwell. 5th edition. Pergamon Press, Oxford.

Goose, D. H. and Lee, G. T. R. (1971). The mode of inheritance of Carabelli's trait. Human Biology, 43, 64-69.

Hrdlicka, A. (1920). Shovel-shaped teeth. American fournal of Physical Anthropology, 3, 429-465.

Kraus, B. S. (1951). Carabelli's anomaly of the maxillary molar teeth: observations on Mexicans and Papago Indians and an interpretation of the inheritance. American fournal of Human Genetics, 3, 348-355.

Turner, C. G. (1969). Microevolutionary interpretations from the dentition. American fournal of Physical Anthropology, 30, 421426. 